\title{
Effects of pressure on the superconducting properties of magnesium diboride
}

\author{
X. J. Chen, H. Zhang, and H.-U. Habermeier \\ Max-Planck-Institut für Festkörperforschung, D-70569 Stuttgart, Germany
}

(Dated: Received 21 May 2001)

\begin{abstract}
We discuss the effects of hydrostatic pressure on the superconducting properties of $\mathrm{MgB}_{2}$ within the framework of Eliashberg theory. By considering the pressure dependences of all parameters appearing in the McMillan formula, we show that the calculated pressure derivative of $T_{c}$ as well as the variation of $T_{c}$ with pressure are in good agreement with recent measurements. The pressure dependences of the energy gap $\Delta_{0}$, the effective interaction strength $N\left(E_{F}\right) v$, the critical magnetic field $H_{c}(0)$, and the electronic specific heat coefficient $\gamma$ are also predicted for this system. A comparison of pressure effect in non-transition elements clearly suggests that $\mathrm{MgB}_{2}$ is an electronphonon mediated superconductor.

PACS numbers: 74.62.Fj, 74.70.Ad
\end{abstract}

\section{INTRODUCTION}

The recent discovery of superconductivity in $\mathrm{MgB}_{2}$ (Ref. 1) has attracted considerable interest in the study of this material, both to understand the mechanism of superconductivity and to explore other properties of $\mathrm{MgB}_{2}$ and related materials. The high transition temperature $T_{c} \approx 40 \mathrm{~K}$ in this material offers other possibility for finding high- $T_{c}$ superconductivity in some binary intermetallic compounds besides cuprates and $C_{60}$-based compounds. Meanwhile, the high critical currents observed in $\mathrm{MgB}_{2}$ thin films and wires 3 reveal that $\mathrm{MgB}_{2}$ belongs to a new class of low-cost, high-performance superconducting materials for magnets and electronic applications.

Measurements of the isotope effect and of the influence of pressure on the transition temperature and critical field of superconductors yield information on the interaction causing superconductivity. Indeed, the pressure (or volume) and the mass number would seem to be the only variables whose effect might be capable of immediate theoretical interpretation. By observing how pressure changes the parameters of the lattice in the normal state and in the superconducting state, and comparing the measurements with the theoretical predictions, one cap test the validity of some theoretical models. Olsen et al. 1 have shown that the volume $(\mathrm{V})$ dependence of effective interaction $N\left(E_{F}\right) v, d \ln N\left(E_{F}\right) v / d \ln V$, can be scaled well with the deviation $\xi$ from the full isotope effect where $\xi$ is defined by $T_{c-1} \times M^{-0.5(1-\xi)}$ in superconducting metals. Bud'ko et al. 0 and Hinks et al. 6 reported a sizeable isotope effect for $\mathrm{B}\left(\alpha_{B}=0.26(3)\right.$ or $\left.0.30(1)\right)$ in newly discovered superconductor $\mathrm{MgB}_{2}$. Although the total isotope coefficient $\alpha=0.32(1)$ (Ref. 6) is smaller than the canonical BCS value of 0.5 , it is the same as that in Cd (Ref. 7). The isotope effect along with other measurements such as inelastic neutron scatteripg, 80 tunneling, 10 NMR (Ref. 11), and specific heat 12 (3) 14 confirmed that $\mathrm{MgB}_{2}$ is an electron-phonon mediated $s$-wave superconductor.

Soon after the discovery of superconductivity in MgP the effect of pressure on $T_{c}$ was studied by two groups 1 . 16 by resistivity or $a c$ susceptibility measurements. Both groups observed a decrease of $T_{c}$ with increasing pressure, with initial pressure derivative $d T_{c} / d P$ of $-0.8 \mathrm{~K} / \mathrm{GPa}$ (Ref. 15) or $-1.6 \mathrm{~K} / \mathrm{GPa}$ (Ref. 16), respectively. Moreover, Monteverde et al. 15 found that the superconductivity is not destroyed by applying high pressure up to $25 \mathrm{GPa}$, at which point $T_{c}$ is as high as $21 \mathrm{~K}$. A somewhat larger $d \mathcal{F}_{\mathrm{g}} / d P$ of $-2.0 \mathrm{~K} / \mathrm{GPa}$ was late reported by Saito et al.17 from high-pressure resistivity measurements. Using a He-gas apparatus, Tomita et al.18 determined a $d T_{c} / d P$ of $-1.11 \mathrm{~K} / \mathrm{GPa}$ under pure hydrostatic pressure conditions. In order to find the reason why the reported values of $d T+d P$ are different among different groups, Lorenz et al. 19 carried out high pressure experiments on $\mathrm{MgB}_{2}$ samples with different $T_{c}$ 's at ambient pressure and different pressure media. $T_{c}$ was found to decrease linearly over the whole pressure range (0-1 GPa). In the He environment, the two samples with the initial $T_{c}=39.2$ and $37.4 \mathrm{~K}$ yield the pressure derivatives of -1.07 and $-1.45 \mathrm{~K} / \mathrm{GPa}$, respectively. The former is obviously very close to that of Temita et al 18 The latter approaches their previous data, 16 which was obtained by using the Fluorinert FC77 as pressure medium. They therefore concluded that the variation in the value of $d T_{c} / d P$ by various groups results from the differences in sample preparation conditions. The value of $d T_{c} / d P \simeq-1.1 \mathrm{~K} / \mathrm{GPa}$ is then confirmed to give the true hydrostatic pressure dependence of $T_{c}$ in $\mathrm{MgB}_{2}$.

Two theoretical models have been tried to describe the systematics of the behavior of $T_{c}$ under pressure in $\mathrm{MgB}_{2}$ Based on the theory of hole superconductivity, Hirsche0 predicted an increased $T_{c}$ with the decrease of B-B intraplane distance under the application of in-plane biaxial pressure. However, this prediction has not been confirmed experimentally yet. No uniaxial pressure measurement was reported due to the extreme difficulty in growing $\mathrm{MgB}_{2}$ single crystal. The experiments of hydrostatic pressure effect on $T_{c}$ do not particularly support this theory provided that no charge transfer between the $\mathrm{Mg}$ and B layers occurs. Alternatimely, the experimental results have heen analyzed 16.18 .21 .22 by using the McMillan formula 23 derived from Eliashberg theory, 24 supporting electron-phonon mediated superconductivity. Inter- 
estingly, Loa and Syassen21 analyzed the pressure effect on $T_{c}$ from McMillan formula on the basis of their calculated elastic and electronic structure data. Assuming that the electron-ion matrix element $I$ is pressure independent, they found that the pressure effect on $T_{c}$ is in good agreement with experimental data by using a lattice Grüneisen parameter $\gamma_{G}=1$. These assumptions, however, deserve some refinements. Recent band structure calculations suggest that $\mathrm{MgB}_{2}$ is a traditional $s p$ metal superconductor.25 26-27 The pressure dependence of $I$ has long been an interesting jspref the research of pressure effect in simple $s p$ metals.28.29.30.31,32 Ziman's calculation of the electron-phonon interaction leads to $\left\langle I^{2}>\propto N\left(E_{F}\right)^{-2}\right.$ at least in the limit of long wavelengths. 33 This then indicated that the consideration of the pressure dependence of $I$ would be important for better understanding the superconducting properties of $\mathrm{MgB}_{2}$ under pressure. On the other hand, it has been found 8 that the choice of lattice Grüneisen parameter $\gamma_{G}$ is crucially important in explaining both the magnitude and the sign of the pressure derivative of $T_{c}$ when using McMillan formula. The value of $\gamma_{G}=1$ in the calculation of Loa and Syassen is obviously lower than those reported recently. 435 The pressure dependence of the effective electron-electron Coulomb repulsion $\mu^{*}$ appearing in the McMillan formula is usually neglected in previous studies due to the assumption of the small change of $\mu^{*}$ compared with that of the electron-phonon coupling parameter $\lambda$ (Ref. 36). However, the magnitude of $\mu^{*}$ is also of interest in connection with the possibility that superconductivity may be destroyed by pressure 3637 It was argued that the pressure dependence of $\mu^{*}$ makes a significant contribution to the behavior of $T_{c}$ under very high pressures and must be handled carefully. 32 . 38 , 39

In this paper we discuss the pressure dependences of some interested superconducting properties in $\mathrm{MgB}_{2}$. The outline of this paper is as follows: In Sec. II we presented a theoretical approach for pressure effects on the superconducting properties in the simple $s p$ metals superconductors. Section III contains the theoretical results obtained and a comparison with experiments for $\mathrm{MgB}_{2}$. We draw conclusions in Sec. IV.

\section{THEORETICAL FORMULATION}

For our purposes, the relation between $T_{c}$ and microscopic parameters is given adequately by the McMillan equation 23

$$
T_{c}=\frac{\Theta_{D}}{1.45} \exp \left[-\frac{1.04(1+\lambda)}{\lambda-\mu^{*}(1+0.62 \lambda)}\right],
$$

which relates $T_{c}$ to the electron-phonon coupling parameter $\lambda$, the Coulomb repulsion strength $\mu^{*}$, and a temperature $\Theta_{D}$ characteristic of the phonons.

Considering the variations of $\Theta_{D}, \lambda$, and $\mu^{*}$ with pressure or volume and introducing parameters $\varphi=$ $\partial \ln \lambda / \partial \ln V$ and $\phi=\partial \ln \mu^{*} / \partial \ln V$, we can get the pressure coefficient of $T_{c}$

$$
\begin{aligned}
\frac{d \ln T_{c}}{d P} & =\frac{\gamma_{G}}{B_{0}}-\frac{1.04 \lambda\left(1+0.38 \mu^{*}\right)}{\left[\lambda-\mu^{*}(1+0.62 \lambda)\right]^{2}} \frac{\varphi}{B_{0}} \\
& +\frac{1.04 \mu^{*}(1+\lambda)(1+0.62 \lambda)}{\left[\lambda-\mu^{*}(1+0.62 \lambda)\right]^{2}} \frac{\phi}{B_{0}},
\end{aligned}
$$

where $B_{0} \equiv 1 / \kappa_{V}=-\partial P / \partial \ln V$ is the bulk modulus and $\Theta_{D}$ is assumed to be proportional to $<\omega^{2}>^{1 / 2}$ and $\gamma_{G}=-\partial \ln <\omega^{2}>^{1 / 2} / \partial \ln V$ being the effective Grüneisen parameter.

It is well known that the usyal BCS result for the energy gap can be expressed by 40

$$
\Delta_{0}=2 \Theta_{D} \exp \left[-\frac{1}{N\left(E_{F}\right) v}\right] .
$$

Where $N\left(E_{F}\right)$ is the electronic density of states at the Fermi energy $E_{F}$ and $v$ is the pairing potential arising from the electron-phonon interaction. If we renormalize the Morel-Anderson result 41 by introducing the renormalization parameter $Z_{n}(0) \equiv 1+\lambda$ into their analysis, the effective interaction strength $N\left(E_{F}\right) v$ can be rewritten as 42

$$
N\left(E_{F}\right) v=\frac{\lambda-\mu^{*}}{1+\lambda} .
$$

The logarithmic volume derivative of $N\left(E_{F}\right) v$ is then given by

$$
\frac{d \ln N\left(E_{F}\right) v}{d \ln V}=\frac{\lambda\left(1+\mu^{*}\right)}{\left(\lambda-\mu^{*}\right)(1+\lambda)} \varphi-\frac{\mu^{*}}{\lambda-\mu^{*}} \phi .
$$

Considering the experimental observations of the pressure dependence of the energy gap of superconductor, 43 we differentiate Eq. (3) with respect to pressure

$$
\frac{d \ln \Delta_{0}}{d P}=\frac{\gamma_{G}}{B_{0}}-\frac{1}{B_{0}}\left[\frac{\lambda\left(1+\mu^{*}\right)}{\left(\lambda-\mu^{*}\right)^{2}} \varphi-\frac{\mu^{*}(1+\lambda)}{\left(\lambda-\mu^{*}\right)^{2}} \phi\right] .
$$

BCS expressign for the critical field $H_{c}$ at absolute zero temperature is $₫$

$$
\frac{H_{c}(0)^{2}}{8 \pi}=2 N\left(E_{F}\right) \omega^{2} \exp \left[-\frac{2}{N\left(E_{F}\right) v}\right] .
$$

Differentiating Eq. (7) with respect to the pressure, one obtains an expression of the pressure coefficient of $H_{c}(0)$,

$$
\frac{d \ln H_{c}(0)}{d P}=\frac{d \ln \Delta_{0}}{d P}-\frac{\gamma_{N}}{2 B_{0}}+\frac{1}{2 B_{0}}
$$

where $\gamma_{N}=\partial \ln N\left(E_{F}\right) / \partial \ln V$.

The expressions for $\gamma_{G}, \varphi$, and $\phi$ can be integrated to give

$$
\begin{aligned}
\Theta_{D}(V) & =\Theta_{D}(0)\left[V / V_{0}\right]^{-\gamma_{G}} \\
\lambda(V) & =\lambda(0)\left[V / V_{0}\right]^{\varphi} \\
\mu^{*}(V) & =\mu^{*}(0)\left[V / V_{0}\right]^{\phi} .
\end{aligned}
$$


Here $V$ and $V_{0}$ are the unit cell volumes under the applied pressure and at ambient pressure, respectively. These two volumes can be related according to the firstorder Murnaghan equation of state $V(P)=V(0)(1+$ $\left.B_{0}^{\prime} P / B_{0}\right)^{-1 / B_{0}^{\prime}}$. The Eq. (9) is then rewritten as

$$
\begin{aligned}
\Theta_{D}(P) & =\Theta_{D}(0)\left[1+\frac{B_{0}^{\prime} P}{B_{0}}\right]^{\gamma_{G} / B_{0}^{\prime}} \\
\lambda(P) & =\lambda(0)\left[1+\frac{B_{0}^{\prime} P}{B_{0}}\right]^{-\varphi / B_{0}^{\prime}} \\
\mu^{*}(P) & =\mu^{*}(0)\left[1+\frac{B_{0}^{\prime} P}{B_{0}}\right]^{-\phi / B_{0}^{\prime}} .
\end{aligned}
$$

From Eqs. (1) and (10) we arrive at the expression for the pressure dependence of $T_{c}$

$$
T_{c}(P)=T_{c}\left[\Theta_{D}(P), \lambda(P), \mu^{*}(P)\right] .
$$

Knowing $B_{0}, B_{0}^{\prime}, \gamma_{G}, \gamma_{N}, \phi$, and $\varphi$, one can evaluate the pressure effects on the superconducting properties, especially the behavior of $T_{c}$ under pressure. $B_{0}$ and $B_{0}^{\prime}$ can be obtained from the compressibility data determined by neutron or synchrotron x-ray diffractions. A direct experimental determination of $\gamma_{G}$ can be made by measuring-electron tunneling 44.45 or inelastic neutron scattering 27 under high pressure. In general, for metals in which different techniques yield similar Grüneisen constants, a good approximation to $\gamma_{G}$ is provided by the room temperature value determined from the Grüneisen equation

$$
\gamma_{G}=\frac{\alpha_{V} V_{m}}{\kappa_{V} C_{p}}
$$

where $\alpha_{V}$ is the volume coefficient of thermal expansion, $V_{m}$ is the molar volume, and $C_{p}$ is the molar heat capacity at constant pressure. The approximation for $\gamma_{G}$ of Slater is derived from the pressure derivative of the bulk modulus 48

$$
\gamma_{G}^{S} \equiv \frac{B_{0}^{\prime}}{2}-\frac{1}{6}=-\frac{2}{3}-\frac{1}{2} \frac{V \partial^{2} P / \partial V^{2}}{\partial P / \partial V} .
$$

The formula for $\mu^{*}$ due to Morel and Anderson 41 used here is

$$
\mu^{*}=\frac{\mu}{1+\mu \ln \left(E_{F} / \omega_{p h}\right)},
$$

with $\mu=0.5 \ln \left[\left(1+a^{2}\right) / a^{2}\right]$ and $a^{2}=\pi e^{2} N\left(E_{F}\right) / k_{F}^{2}$, from which we evaluate the volume dependence of $\mu^{*}$ as

$$
\phi=\mu^{*}\left[\frac{2}{3}-\gamma_{G}-\frac{1-e^{-2 \mu}}{2 \mu^{2}}\left(\gamma_{N}+\frac{2}{3}\right)\right] .
$$

Here the variation of $k_{F}$ with volume has been calculated from the fundamental definition $k_{F}=\left(3 \pi^{2} Z / V\right)^{1 / 3}$ with $Z$ the valency. Unfortunately, to the best of our knowledge $\gamma_{N}$ has never measured directly for any superconductor in the case of free electron gas it would have a value of $2 / 3$. Using the expression given by Migdal 9 for the electronic specific heat coefficient $\gamma$ one obtains for the electronic Grüneisen parameter

$$
\gamma_{e}=\frac{\partial \ln \gamma}{\partial \ln V}=\gamma_{N}+\frac{\lambda}{1+\lambda} \varphi .
$$

The electronic Grüneisen parameter $\gamma_{e}$ is usuallydeduced from measurements through the simple relation 5

$$
\gamma_{e}=\frac{\alpha_{e} V_{m}}{\kappa_{V} C_{e}}
$$

Here $\alpha_{e}$ is the contribution to the expansion coefficient from the electrons at lower temperatures and $C_{e}$ is the electronic heat capacity. A theoretical estimate of $\gamma_{e}$ can also be given from the measurement of the volume dependence of the orbital susceptibility 51 or from bandstructure considerations 52

The electron-phonon coupling parameter $\lambda$ can be expressed as

$$
\lambda=\frac{N\left(E_{F}\right)<I^{2}>}{M<\omega^{2}>} \equiv \frac{\eta}{M<\omega^{2}>},
$$

where $<I^{2}>$ is the mean-square electron-ion matrix element and $M$ the ionic mass. The McMillan-Hopfield parameter $\eta$ (or $N\left(E_{F}\right)<I^{2}>$ ) has been regarded as a local "chemical" property of an atom in a crystal. Allen and Dynes 53 pointed out that $\eta$ is the most significant single parameter in understanding the origin of the high $T_{c}$ of conventional superconductors. For strong coupling systems, variation in $\eta$ is more important than variation of $\left\langle\omega^{2}\right\rangle$ in causing $T_{c}$ to change. Softening of $\left\langle\omega^{2}\right\rangle$ often does enhance $T_{c}$, but very high $T_{c}$ should be caused more by large $\eta$ than by small $\left\langle\omega^{2}\right\rangle$.

The logarithmic volume derivative of $\lambda, \varphi$, is then obtained

$$
\varphi=\frac{\partial \ln \eta}{\partial \ln V}+2 \gamma_{G} \equiv S+2 \gamma_{G}
$$

In order to understand how the electronic contribution $\eta=N\left(E_{F}\right)<I^{2}>$ yaries with volume, we use the Gaspari-Gyoriffy theory 54 for $\eta$, i.e.,

$$
\eta=\frac{k_{F}^{2}}{\pi^{2} N\left(E_{F}\right)} \sum_{l} \frac{2(l+1) \sin ^{2}\left(\delta_{l+1}-\delta_{l}\right) N_{l} N_{l+1}}{N_{l}^{1} N_{l+1}^{1}}
$$

where $N_{l}$ is the $l$ th angular momentum component of the density of states, $N_{l}^{1}$ is the $l$ th component of the single scatterer density of states evaluated at $E_{F}$, and $\delta_{l}$ the phase shift.

For simple metals, the scatterers are assumed to be weak. We can take $N_{l}=N_{l}^{1}$ and approximate $\sin ^{2}\left(\delta_{l+1}-\right.$ $\left.\delta_{l}\right)$ by $\left(\delta_{l+1}-\delta_{l}\right)^{2}$. Eq. (20) is rewritten as

$$
\eta=\frac{k_{F}^{2}}{\pi^{2} N\left(E_{F}\right)} \sum_{l} 2(l+1)\left(\delta_{l+1}-\delta_{l}\right)^{2} .
$$


This expression is identical to the pseudopotential formula of McMillan.23.55 Assuming that the phase shift $\delta_{l}$ does not vary very much under pressure for simple $s p$ superconductors, we then obtain

$$
S=-\gamma_{N}-\frac{2}{3}
$$

The form in $\mathrm{E}$ (22) is the same as that of Baryakhtar and Makarov, 56 who used the constant of the electronphonon interaction of Fröhlich and Mitra.57 The expression is an improvement over the expressions of $S=0$ and $S=-4 / 3$ obtained by Olsen et al 58 and Seiden, 36 respectively. It is interesting to notice that substitution of $\gamma_{N}=2 / 3$ into Eq. (22) yields $S=-4 / 3$. Eq. (22) reduces to the expression of Seiden, 6 who modified McMillan's expression for $\lambda$ somewhat by considering the effects of a real lattice spectrum as opposed to the Jellium model. Since the electronic Grüneisen parameter $\gamma_{e}$ usually varies among different metals even in the simple non-transition elements, 5052 we believe that Eq. (22) should provide a more reasonable value of $S$ compared with Seiden's formula.

\section{RESULTS AND DISCUSSION}

Using the experimental value of $T_{c}=39.25 \mathrm{~K}$ (Refs. 18 , 19), and the theoretical estimates of $\lambda=0.87$ and $\mu^{*}=0.10$ (Ref. 27), we got $\Theta_{D}=860 \mathrm{~K}$ from Eq. (1) for $\mathrm{MgB}_{2}$. We believe that all these parameters, which will enter our calculations, are reliable. For example, the inelastic neutron scattering measurement 9 provide an estimate of $\lambda \sim 0.9$, which is close to that we used. The calculated value of $\Theta_{D}=860 \mathrm{~K}$ is in the range from 746 to $1050 \mathrm{~K}$ determined from the specific heat measurements.2. 2.3 .14

We took the structural parameters $B_{0}=147.2 \mathrm{GPa}$ and $B_{0}^{\prime}=4$ from the measurements under the pure hydrostatic pressures up to $0.62 \mathrm{GPa}$ (Ref. 59) and under high pressures up to $15 \mathrm{GPa}$ (Refs. 22 35), respectively. To our knowledge no inelastic neutron scattering or tunneling data exist for $\mathrm{MgB}_{2}$ under hydrostatic pressure. We have to use Eq. (12) or (13) for estimating the lattice Grüneisen parameter $\gamma_{G}$. The measurements of heat capacity 60 give a $C_{p}$ of $47.80 \mathrm{~J} /(\mathrm{K} \mathrm{mol})$ at $T=298.16$ K. $V_{m}=1.75 \times 10^{-5} \mathrm{~m}^{3} / \mathrm{mol}, \kappa_{V}=6.79 \times 10^{-12} \mathrm{~Pa}^{-1}$, and $\alpha_{V}=2.22 \times 10^{-5} \mathrm{~K}^{-1}$ can be drawn from the neutron diffraction data. 59 We therefore obtained $\gamma_{G}=1.2$ by using Eq. (12). Based on the first-order Murnaghan equation for $V(P)$ and the Slater expression of Eq. (13), we got a somewhat larger $\gamma_{G}$ of 1.83 compared to that from Eq. (12). For most simple metals, there is no much difference between the room temperature lattice Grüneisen parameter given through Eq. (12) and the Slater relation 36.61 It was found that the Slater expression usually san yield the reasonable values of $\gamma_{G}$ for most metals 62 The only uncertainty entering Eq. (12) in our calculation comes from the indirect measurements of the linear coefficients of thermal expansion. 5 Roundy

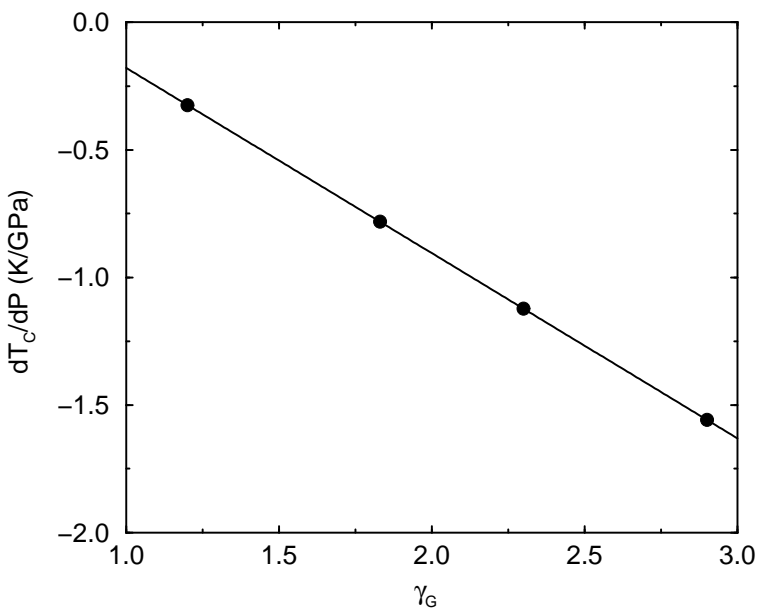

FIG. 1: Pressure derivative of $T_{c}$ as a function of the lattice Grüneisen parameter $\gamma_{G}$ in $\mathrm{MgB}_{2}$. The circles show the calculation from the four different values of $\gamma_{G}=1.2,1.83,2.3$, and 2.9 .

et al 34 reported a value of $\gamma_{G} \approx 2.3$ from ab initio calculations, which is close to our calculated $\gamma_{G}^{S}$ according to Eq. (13). Meanwhile, Goncharov et al .35 determined a large $E_{2 g}$ mode Grüneisen parameter of $2.9 \pm 0.3$ from the measurements of Raman spectra under pressure. This value is obviously larger than those derived from Eqs. (12) and (13).

In the calculations of electronic density of states, Loa and Syassan21 found that $N\left(E_{F}\right)$ decreases with pressure at a rate of $d \ln N\left(E_{F}\right) / d P=-3.1 \times 10^{-3} \mathrm{GPa}^{-1}$. Combining this calculated value and experimental value of $B_{0}$, we got $\gamma_{N}=0.46$. The volume dependence of $\mu^{*}$ is then derived from (15) once having the values of $\gamma_{G}$ and $\mu$. For simple $s p$ metals, $a^{2}$ has a typical value of 0.4 (Ref. 41), which yields $\mu=0.63$. The volume dependence of $\lambda, \varphi$, is therefore determined from Eqs. (19) and (22). Using $\gamma_{N}=0.46$, we have $S=-1.13$ for $\mathrm{MgB}_{2}$, which is smaller in magnitude than $-4 / 3$ in Seiden's formula for simple metals. 36 For the transition metals, Hopfield 63 commented that $S$ is a relatively constant quantity with a value of about -3.5 . The values of $S=-3.5 \sim-3.1$ obtained by inverting the measured $d T_{c} / d P$ for $\mathrm{YNi}_{2-x} \mathrm{M}_{x} \mathrm{~B}_{2} \mathrm{C}$ $(\mathrm{M}=\mathrm{Co}$ and $\mathrm{Cu})$ (Ref. 64) are comparable to that of the transition metals, but are larger in magnitude compared to that of $\mathrm{MgB}_{2}$.

With the parameters determined above, we have calculated the pressure derivatives of $T_{c}$ for $\mathrm{MgB}_{2}$ by using Eq. (2). In Fig. 1 we plotted $d T_{c} / d P$ as a function of $\gamma_{G}$ in the interested range. It is interesting to note that $\gamma_{G}$ plays a predominant role for the pressure effect of $T_{c}$. For the four different $\gamma_{G}$ 's considered here $d T_{c} / d P$ are negative. The values obtained from $\gamma_{G}=1.83$ and 2.3 are -0.78 and $-1.12 \mathrm{~K} / \mathrm{GPa}$, respectively. These are close to the hydrostatic pressure value of $-1.1 \mathrm{~K} / \mathrm{GPa}$ 


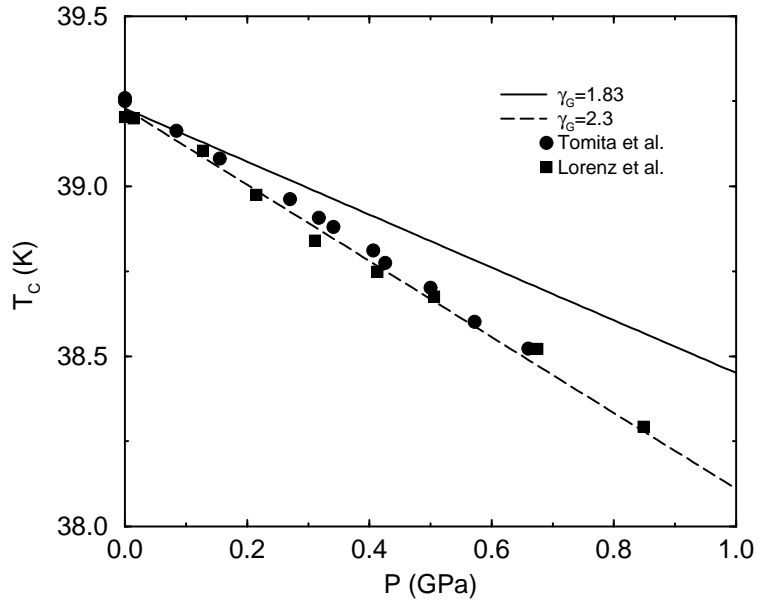

FIG. 2: Variation of $T_{c}$ with pressure in the region of 0 to 1.0 GPa of $\mathrm{MgB}_{2}$ for $\gamma_{G}=1.83$ and 2.3, respectively. The circles and squares represent the hydrostatic pressme experimental datw taken from the works of Tomita et al 18 and Lorenz et al. 19 respectively.

(Refs. 18, 19). Thus the hydrostatic pressure results can be reproduced in terms of our present model by using the values of $\gamma_{G}$ obtained from either the Slater relation or $a b$ initio calculation. It is difficult to obtain the measured results by using $\gamma_{G}=1$ as suggested by Loa and Syassen.21 We noticed that a $\gamma_{G}$ of 2.27 is necessary so as to account for the pressure effect on $T_{c}$ for $\mathrm{MgB}_{2}$. As emphasized above, all quantities entering Eq. (12) are experimental values and only $\alpha_{V}$ was taken from indirect measurements. Thus it is highly expected to operate the thermal expansion measurement to yield a direct $\alpha_{V}$. The present results indicate that the range from $\gamma_{G}=1.83$ to 2.3 should cover the reasonable choices for the lattice Grüneisen parameters.

To verify these results, and also to study the behavior of $T_{c}$ as a function of pressure, we have performed explicit calculation based on Eq. (11). The theoretical results in the pressure range from 0 to $1.0 \mathrm{GPa}$ are shown in Fig. 2. The experimental data points of Tomita et al.18 and Lorenz et al 19 measured under hydrostatic pressure conditions are also plotted for comparison. It is clearly seen that our calculations agree well with the experiments.

In Fig. 3 we presented the calculated results as mell as the experimental data points of Monteverde et al 15 and Deemyad et al 65 measured in the relatively high pressure region. Here we assume that phase transitions of all kinds do not occur under pressure range that we consider. We noticed that the experimental data points of Peemyad et al.65 and the sample 4 of Monteverde et al.15 are situated well between the two theoretical curves calculated by using $\gamma_{G}=1.83$ and 2.3 , respectively. Interestingly, the agreement between our theoretical curve calculated by using $\gamma_{G}=1.83$ and the experimental data points of other samples of Monteverde et al. .5 is seen to be reasonable,

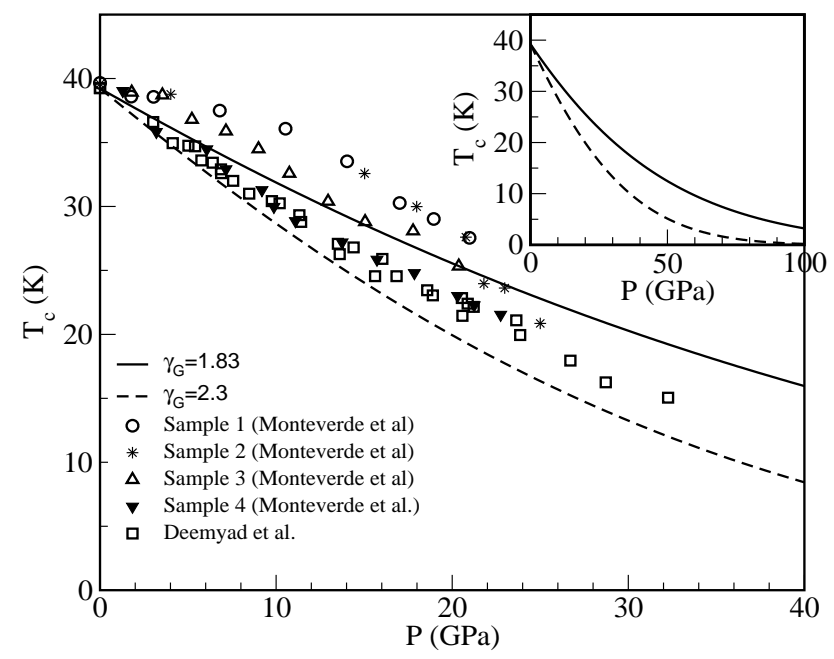

FIG. 3: Pressure dependence of the transition temperature in $\mathrm{MgB}_{2}$ up to $40 \mathrm{GP}_{\text {merimental data }}$ Experom the works of Monteverde et al.5 and Deemyad et al.55 The inset is a calculation of $T_{c}$ under pressure up to $100 \mathrm{GPa}$.

although there are some scatters among different samples and the reason is not clear. Furthermore, although the pressure measurements are limited to the region below $33 \mathrm{GPa}$, it is seen from the inset of Fig. 3 that Eq. (11) continues to describe the pressure dependence of $T_{c}$ as high as $100 \mathrm{GPa}$. Even at this point, the superconductivity is not destroyed by pressure in newly discovered superconductor $\mathrm{MgB}_{2}$. There was a discrepancion whether pressure can destroy superconductivity.36.37.66.67 However, our resplts support the conclusion of Olsen and collaborators 3760 that the possibility of destruction of superconductivity by the application of sufficiently high pressure most likely does not exist. It follows from the comparison of Figs. 2 and 3 that the the pressure effect on $T_{c}$ indeed provides a support to the electron-phonon mediated superconductivity in $\mathrm{MgB}_{2}$.

In Fig. 4 we presented the normalized $\lambda$ and $\mu^{*}$ as a function of pressure up to $30 \mathrm{GPa}$, calculated from Eq. (10) by using $\gamma_{G}=2.3$. The Coulomb pseudopotential $\mu^{*}$ increases slightly with pressure. Whereas $\lambda$ changes significantly with pressure. The contribution from $\mu^{*}(\mathrm{P})$ to the variation of $T_{c}$ with pressure is much less important than that of $\lambda(\mathrm{P})$. Thus in the range from 0 to $30 \mathrm{GPa}$ the pressure effect of $T_{c}$ for $\mathrm{MgB}_{2}$ is dominated by the competition of $\lambda$ and $\Theta_{D}\left(\right.$ or $\left.<\omega^{2}>^{1 / 2}\right)$.

TABLE I: Pressure dependences of superconducting state parameters in $\mathrm{MgB}_{2}$. The units of $d \ln X / d P\left(\mathrm{X}=T_{c}, \Delta_{0}\right.$, are $\left.H_{c}(0)\right)$ are in $10^{-2} \mathrm{GPa}^{-1}$.

\begin{tabular}{cccccc}
\hline \hline$\gamma_{G}$ & $\gamma_{e}$ & $\frac{d \ln N\left(E_{F}\right) v}{d \ln V}$ & $\frac{d \ln T_{c}}{d P}$ & $\frac{d \ln \Delta_{0}}{d P}$ & $\frac{d \ln H_{c}(0)}{d P}$ \\
\hline 1.83 & 1.64 & 1.71 & -1.99 & -1.58 & -1.39 \\
2.3 & 2.07 & 2.34 & -2.86 & -2.30 & -2.11 \\
\hline \hline
\end{tabular}




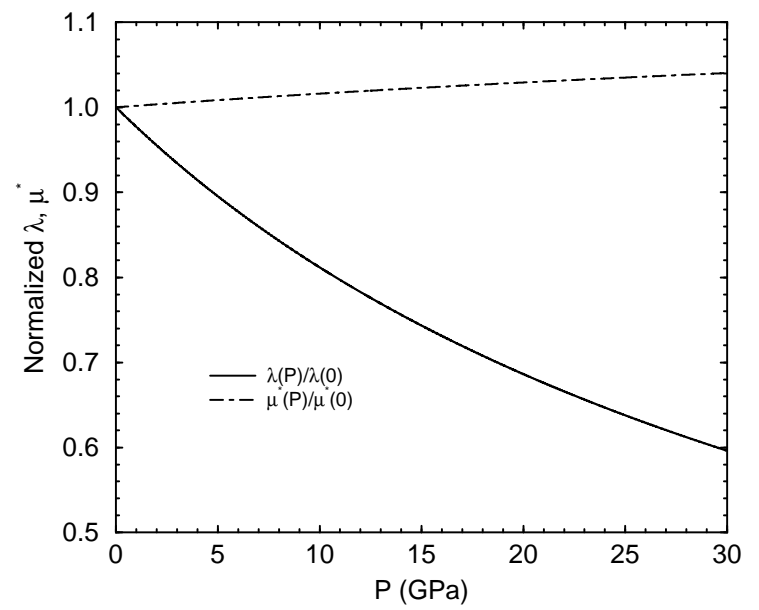

FIG. 4: Pressure dependence of normalized Coulomb $\left(\mu^{*}\right)$ and electron-phonon coupling $(\lambda)$ coupling strengths in $\mathrm{MgB}_{2}$ calculated by using $\gamma_{G}=2.3$.

Table I contains the calculated values of pressure dependences of superconducting parameters for $\mathrm{MgB}_{2}$ from Eqs. (5), (6), (8) and (16) by using $\gamma_{G}=1.83$ and $\gamma_{G}=2.3$, respectively. The reliable values of $\gamma_{e}$ is readily determined using Eqs. (16), (19), and (22). We obtained $\gamma_{e}=1.64$ and 2.07 for $\mathrm{MgB}_{2}$, which are close to $\gamma_{e}=1.7$ for $\mathrm{Pb}$ and for $\mathrm{Sn}, \gamma_{e}=2.0$ for $\mathrm{Al}$ (Ref. 67). The negative sign for $d \ln H_{c}(0) / d P$ predicted for $\mathrm{MgB}_{2}$ is in agreement with the measurements for all simple elements superconductors with the exception of thallimm. 88 For the simple $s p$ metals superconductors, Rohrer 69 has demonstrated that $d \ln N\left(E_{F}\right) v / d \ln V$ must have approximately a value of 2.0 . However, it was realized 68 that the transition metals fail to show such simple behavior. Our estimated $d \ln N\left(E_{F}\right) v / d \ln V=1.71$ and 2.34 for $\mathrm{MgB}_{2}$ are comparable to those obtained for simple $s p$ metals superconductprs 6860 Early measurements for most simple metals 2 . 43 . 46 show that there is difference between the quantities in $d \ln \Delta_{0} / d P$ and $d \ln T_{c} / d P$. This can be understogd with the aid of the results by Geilikman and Kresin, 70 that is, $2 \Delta_{0} / k_{B} T_{c}=3.52[1+$ $5.3\left(T_{c} / \omega_{p h}\right)^{2} \ln \omega_{p h} / T_{c}$ ]. The calculated data of $\mathrm{MgB}_{2}$ listed in Table I make it possible to support this theory. Since the phonon spectrum shifts under pressure, it follows that for all superconductors with $2 \Delta_{0} / k_{B} T_{c}>3.52$ a change of $2 \Delta_{0} / k_{B} T_{c}$ under pressure can be expected. It is interesting from the viewpoint of experiment to investigate the tunnel characteristics of $\mathrm{MgB}_{2}$ under hydrostatic pressure.

Figure 5 is a plot of $d \ln N\left(E_{F}\right) v / d \ln V$ versus the deviation $\xi$ from the full isotope effect for nine simple $s p$ metals superconductors as well as $\mathrm{MgB}_{2}$. The experimental values of $d \ln N\left(E_{F}\right) v / d \ln V$ for simple metals are chosen from the work of Olsen, Andres, and Geballe. 58 The experimental results for isotope effect exponent $\alpha$

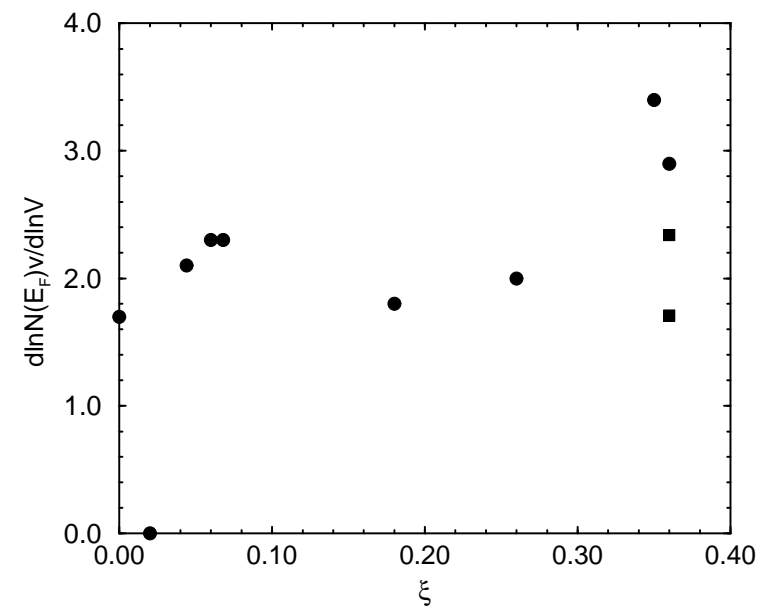

FIG. 5: Relation between the logarithmic volume derivative of $N\left(E_{F}\right) v$ and the deviation $\xi$ from the full isotope effect exponent $\alpha=0.5(1-\xi)$ in nine simple $s p$ metals superconductors and $\mathrm{MgB}_{2}$. The two squares are the values for $\mathrm{MgB}_{2}$.

and its deviation $\xi$ are taken from the works in Refs. [ 6, $71,72,73,74,, 75$. . There are no experimental data available for $\mathrm{Al}$ and In now, we took the calculated results from Leavens and Carbotte. 12 We summarized the re results for simple $s p$ metals in Table II. Olsen et al $\mathbf{1}$ suggested that $d \ln N\left(E_{F}\right) v / d \ln V$ is related to the isotope effect exponent $\alpha$ in metals superconductors. As seen from Fig. 5 the relation between $d \ln N\left(E_{F}\right) v / d \ln V$ and $\xi$ is not very clear when more data included. An isotope effect $\alpha=0.32(1)$ (Ref. 6) in $\mathrm{MgB}_{2}$ is consistent with what appears to be a systematic variation of $\alpha$ across the nontransition elements. It is well known that deviations of the isotope effect exponent from $1 / 2$ are a measure of the relative strengths of the Coulomb and phonon-mediated electron-electron interactions. It is indicated, from the compared values of $\xi$ for $\mathrm{MgB}_{2}$ (Ref. 6) with $\mathrm{Zn}$ (Ref.

TABLE II: Experimental values of $d \ln N\left(E_{F}\right) v / d \ln V$, isotope effect exponent $\alpha$ and its derivative $\xi=1-2 \alpha$ in nine simple $s p$ metals superconductors.

\begin{tabular}{ccccc}
\hline \hline Element & $\mathrm{Z}$ & $\frac{d \ln N\left(E_{F}\right) v}{d \ln V}$ & $\alpha$ & $\xi$ \\
\hline $\mathrm{Zn}$ & 2 & 2.0 & 0.37 & 0.26 \\
$\mathrm{Cd}$ & 2 & 2.9 & 0.32 & 0.36 \\
$\mathrm{Hg}(\alpha)$ & 2 & 1.7 & 0.50 & 0 \\
$\mathrm{Al}$ & 3 & 3.4 & 0.325 & 0.35 \\
$\mathrm{Ga}$ & 3 & 1.8 & 0.41 & 0.18 \\
$\mathrm{In}$ & 3 & 2.3 & 0.466 & 0.068 \\
$\mathrm{Tl}$ & 3 & 0 & 0.49 & 0.02 \\
$\mathrm{Sn}$ & 4 & 2.3 & 0.47 & 0.06 \\
$\mathrm{~Pb}$ & 4 & 2.1 & 0.478 & 0.044 \\
\hline \hline
\end{tabular}


73), $\mathrm{Cd}$ (Ref. 7), and $\mathrm{Al}$ (Ref. 42), that $\mathrm{MgB}_{2}$ should be a medium coupling superconductor.

\section{SUMMARY AND CONCLUSIONS}

The major conclusions given by present investigation can be summarized as follows:

(i) A simple expression was derived for the pressure dependences of superconducting properties in simple $s p$ superconductor on the basis of McMillan equation. The logarithmic volume derivatives of $\lambda, \mu^{*}$, and $\Theta_{D}$ can be self-consistently determined from experiments and theories. We gave an expression for $\varphi$ from the theory of Gaspari and Gyorffy. 54 The theory of Morel and Anderson 11 was used to obtain $\phi$, which makes it possible to investigate the pressure dependence of $\mu^{*}$. Neglecting the pressure dependence of $\mu^{*}$, the present theoretical model can be reduced to the two popular models of Seiden 36 and Baryakhtar and Makarov56 when taking $\gamma_{N}=2 / 3$ and neglecting the direct electron-electron interaction, respectively. Furthermore, we obtained an explicit expression for the change of $T_{c}$ as a function of pressure with the help of Murnaghan equation. The present model enables us to study the pressure behaviors of some interested superconducting parameters such as the zero temperature energy gap $\Delta_{0}$, the critical field at absolute zero temperature $H_{c}(0)$, the effective interaction strength $N\left(E_{F}\right) v$, and the electronic specific heat coefficient $\gamma$.

(ii) We investigated the pressure effects on superconducting properties in the newly discovered superconductor $\mathrm{MgB}_{2}$ using our simple approach. It was found that the hydrostatic pressure derivative of $T_{c}$ can be reproduced by using the values of $\gamma_{G}$ obtained from either the Slater relation or ab initio calculation. The calculated $d \ln N\left(E_{F}\right) v / d \ln V \approx 2.0$ in $\mathrm{MgB}_{2}$ is close to those obtained in simple $s p$ superconductors. The quantitative agreement for the variation of $T_{c}$ with pressure in the low pressure region as well as high pressure region is very good when comparing our theoretical results with experimental data measured by three groups. The predicted values of $d \ln H_{c}(0) / d P, d \ln \Delta_{0} / d P$, and $\gamma_{e}$ are also comparable to those in simple $s p$ metals superconductors. All these characteristic pressure behaviors allow us to conclude that $\mathrm{MgB}_{2}$ should be a simple electronphonon mediated $s p$ superconductor and the mechanism in simple $s p$ metals superconductors is also responsible for the superconductivity in $\mathrm{MgB}_{2}$.

\section{Acknowledgments}

The authors acknowledge useful discussions with $\mathrm{O}$. K. Andersen, O. Jepsen, Y. Kong, R. K. Kremer, and K. Syassen. We are indebted to J. S. Schilling and J. D. Jorgensen for allowing us to use their experimental data prior to publication as well as their valuable comments on the manuscript. XJC thanks the MPG for financial support.

Note added.- After submission of this manuscript, the authors have learned that the superconductivity is not destroyed up to $44 \mathrm{GPa}$ where $T_{c}$ is still as high as 12 K.6 The intrinsic $d T_{c} / d P \approx-1.1 \mathrm{~K} / \mathrm{GPa}$ under hydrostatic pressure conditions has recently been reported by other three groups 76.77.:88 We also have-learned a possible explanation given by Tissen et al. 99 for the large $-d T_{c} / d P$ observed in the low $T_{c}=37.4 \pm 0.1$ samples.
1 J. Nagamatsu, N. Nakagawa, T. Muranaka, Y. Zenitanl, and J. Akimitsu, Nature (London) 410, 63 (2001).

2 C. B. Eom, M. K. Lee, J. H. Choi, L. J. Belenky, X. Song, L. D. Cooley, M. T. Naus, S. Patnaik, J. Jiang, M. Rikel, A. Polyanskii, A. Gurevich, X. Y. Cai, S. D. Bu, S. E. Babcock, E. E. Hellstrom, D. C. Larbalestier, N. Rogado, K. A. Regan, M. A. Hayward, T. He, J. S. Slusky, K. Inumaru, M. K. Haas, and R. J. Cava, Nature (London) 411, 558 (2001)

3 S. Jin, H. Mavoori, C. Bower, and R. B. van Dover, Nature (London) 411, 563 (2001).

4 J. L. Olsen, E. Bucher, M. Levy, J. Muller, E. Corenzwit, and T. Geballe, Rev. Mod. Phys. 36, 168 (1964).

5 S. L. Bud'ko, G. Lapertot, C. Petrovic, C. E. Cunningham, N. Anderson, and P. C. Canfield, Phys. Rev. Lett. 86, 1877 (2001).

6 D. G. Hinks, H. Claus, and J. D. Jorgensen, Nature (London), 411, 457 (2001).

7 C. Palmy, Phys. Lett. A 29, 373 (1969)

8 T. J. Sato, K. Shibata, and Y. Takano, cond-mat/0102468 (2001).

9 R. Osborn, E. A. Goremychkin, A. I. Kolesnikov, and D.
G. Hinks, Phys. Rev. Lett. 87, 017005 (2001).

10 G. Karapetrov, M. Iavarone, W. K. Kwok, G. W. Crabtree, and D. G. Hinks, Phys. Rev. Lett. 86, 4374 (2001).

11 H. Kotegawa, K. Ishida, Y. Kitaoka, T. Muranaka, and J. Akimitsu, Phys. Rev. Lett. 87, 0127001 (2001).

12 R. K. Kremer, B. J. Gibson, and K. Ahn, condmat/0102432 (2001).

Ch. Wälti, E. Felder, C. Degen, R. Monnier, B. Delley, and H. R. Ott, Phys. Rev. B 64, 172515 (2001).

${ }^{14}$ F. Bouquet, R. A. Fisher, N. E. Phillips, D. G. Hinks, and J. D. Jorgensen, Phys. Rev. Lett. 87, 047001 (2001).

15 M. Monteverde, M. Núñez-Regueiro, N. Rogado, K. A. Regan, M. A. Hayward, T. He, S. M. Loureiro, and R. J. Cava, Science 292, 75 (2001).

16 B. Lorenz, R. L. Meng, and C. W. Chu, Phys. Rev. B 64, 012507 (2001).

17 E. Saito, T. Taknenobu, T. Ito, Y. Iwasa, K. Prassides, and T. Arima, J. Phys.: Condens. Matter 13, L267 (2001).

18 T. Tomita, J. J. Hamlin, J. S. Schilling, D. G. Hinks, and J. D. Jorgensen, Phys. Rev. B 64, 092505 (2001).

19 B. Lorenz, R. L. Meng, and C. W. Chu, cond-mat/0104303 (2001). 
20 J. E. Hirsch, Phys. Lett. A 282, 392 (2001).

21 I. Loa and K. Syassen, Solid State Commun. 118, 279 (2001).

22 T. Vogt, G. Schneider, J. A. Hriljac, G. Yang, and J. S. Abell, Phys. Rev. B 63, 220505 (2001).

23 W. L. McMillan, Phys. Rev. 167, 331 (1968).

${ }^{24}$ G. M. Eliashberg, Sov. Phys. JETP 11, 696 (1960).

25 J. Kortus, I. I. Mazin, K. D. Belashenko, V. P. Antropov, and L. L. Boyer, Phys. Rev. Lett. 86, 4656 (2001).

26 J. M. An and W. E. Pickett, Phys. Rev. Lett. 86, 4366 (2001).

27 Y. Kong, O. V. Dolgov, O. Jepsen, and O. K. Andersen, Phys. Rev. B 64, 020501 (2001).

28 R. Hodd, Phys. Rev. 180, 530 (1969).

29 P. N. Trofimenkoff and J. P. Carbotte, Phys. Rev. B 1, 1136 (1970).

30 M. A. Coulthard, J. Phys. F: Metal Phys. 1, 195 (1971).

31 H. R. Ott and R. S. Sorbello, J. Low Temp. Phys. 14, 73 (1974).

32 M. M. Dacorogna, M. L. Cohen, and P. K. Lam, Phys. Rev. B 34, 4865 (1986).

33 J. M. Ziman, Electrons and Phonos (Clarendon Press, Oxford, 1960).

34 D. Roundy, H. J. Choi, H. Sun, S. G. Louie, and M. L. Cohen (unpublished).

35 A. F. Goncharov, V. V. Struzhkin, E. Gregoryanz, J. Hu, R. J. Hemley, H.-K. Mao, G. Lapertot, S. L. Bun'ko, and P. C. Canfield, Phys. Rev. B 64, 100509 (2001).

36 P. E. Seiden, Phys. Rev. 179, 458 (1969).

37 R. I. Boughton, G. Brändli, J. L. Olsen, and C. Palmy, Helv. Phys. Acta 42, 587 (1969).

38 D. U. Gubser and A. W. Webb, Phys. Rev. Lett. 35, 104 (1975).

39 D. A. Papaconstantopoulos and B. M. Klein, Physica B 107, 725 (1981).

40 J. Bardeen, L. N. Cooper, and J. R. Schrieffer, Phys. Rev. 108, 1175 (1957).

41 P. Morel and P. W. Anderson, Phys. Rev. 125, 1263 (1962).

42 C. R. Leavens and J. P. Carbotte, Can. J. Phys. 49, 724 (1971).

43 J. P. Franck and W. J. Keeler, Phys. Rev. Lett. 20, 379 (1968).

44 J. P. Franck and W. J. Keeler, Phys. Lett. A 25, 624 (1967).

45 J. Yamashita and S. Asano, Jpn. J. Phys. Soc. 29, 264 (1970).

46 N. V. Zavaritskii, E. S. Itskevich, and A. N. Voronovskii, Spv. Phys. JETP 33, 762 (1971).

47 R. Lechner and G. Quittner, Phys. Rev. Lett. 17, 1259 (1966).

48 J. C. Slater, Introduction to Chemical Physics (McGrawHill, New York, 1939).

49 A. B. Migdal, Sov. Phys. JETP 7, 996 (1958).

${ }^{50}$ T. H. K. Barron, J. G. Collins, and G. K. White, Adv. Phys. 29, 609 (1980).

51 E. Fawcett and G. K. White, J. Appl. Phys. 39, 576 (1968).
52 G. C. Fletcher and M. Yahaya, J. Phys. F: Metal Phys. 9, 1529 (1979).

53 P. B. Allen and R. C. Dynes, Phys. Rev. B 12, 905 (1975).

54 G. D. Gaspari and B. L. Gyorffy, Phys. Rev. Lett. 28, 801 (1972).

55 R. Evans, G. O. Gaspari, and B. L. Gyorffy, J. Phys. F: Metal Phys. 3, 39 (1973).

56 V. G. Baryakhtar and V. I. Makarov, Sov. Phys. JETP 22, 1320 (1966).

57 H. Fröhlich and T. K. Mitra, Proc. Phys. Soc. 1, 544 (1968).

58 J. L. Olsen, K. Andres, and T. H. Gebelle, Phys. Lett. A 26, 239 (1968).

59 J. D. Jorgensen, D. G. Hinks, and S. Short, Phys. Rev. B 63, 224522 (2001).

60 R. M. Swift and D. White, J. Am. Chem. Soc. 79, 3641 (1957).

61 K. A. Gschneidner, Jr., in Solid State Physics, edited by F. Seitz and D. Turnbull (Academic Press Inc., New York, 1964), Vol. 16.

62 V. L. Moruzzi, J. F. Fanak, and K. Schwarz, Phys. Rev. B 37, 790 (1988).

63 J. J. Hopfield, Physica 55, 41 (1971).

64 C. Looney, A. K. Gangopadhyay, and A.-K. Klehe, and J. S. Schilling, Physica C 252, 199 (1995).

65 S. Deemyad, J. S. Schilling, J. D. Jorgensen, and D. G. Hinks (unpublished).

66 J. L. Olsen and H. Rohrer, Helv. Phys. Acta 33, 872 (1960); M. Levy and J. L. Olsen, Solid State Commun. 2, 137 (1964).

67 T. F. Smith and C. W. Chu, Phys. Rev. 159, 353 (1967).

${ }^{68}$ M. Levy and J. S. Olsen, in Physics of High Pressures and the Condensed Phase, edited by A. Van Itterbeek (NorthHolland Pub., Amsterdam, 1965).

69 H. Rohrer, Helv. Phys. Acta 33, 675 (1960).

70 B. T. Geilikman and V. Z. Kresin, Sov. Phys. Solid State 7, 2659 (1966).

71 E. Maxwell, Phys. Today 5, No. 12, 14 (1952).

72 R. R. Hake, D. E. Mapother, and D. L. Decker, Phys. Rev. 112, 1522 (1958).

73 R. E. Fassnacht and J. R. Dillinger, Phys. Rev. 164, 565 (1967).

74 R. E. Fassnacht and J. R. Dillinger, Phys. Lett. A 28, 741 (1969).

75 R. Meservey and B. B. Schwartz, in Superconductivity, edited by R. D. Parks (M. Dekker Inc., New York, 1969).

76 V. V. Struzhkin, A. F. Goncharov, R. J. Hemley, H.-K. Mao, G. Lapertot, S. L. Bud'ko, and P. C. Canfield, condmat/0106576 (2001).

77 S. I. Schlachter, W. H. Fietz, K. Grube, and W. Goldacker, cond-mat/0107205 (2001).

78 J. Tang, L. C. Qin, A. Matsushita, Y. Takano, K. Togano, H. Kito, and H. Ihara, Phys. Rev. B 64, 132509 (2001).

79 V. G. Tissen, M. V. Nefedova, M. N. Kolesnikov, and M. P. Kulakov, Physica C 363, 194 (2001). 